\title{
Effects of systemic administration of ciliary neurotrophic factor on Bax and Bcl-2 proteins in the lumbar spinal cord of neonatal rats after sciatic nerve transection
}

\author{
A.C.S. Rezende ${ }^{1 *}$, A.S. Vieira ${ }^{1 *}$, F. Rogério ${ }^{1}$, L.F. Rezende ${ }^{1}$, A.C. Boschero ${ }^{1}$, A. Negro ${ }^{2}$ \\ and F. Langone ${ }^{1}$ \\ 1Departamento de Fisiologia e Biofísica, Instituto de Biologia, Universidade Estadual de Campinas, \\ Campinas, SP, Brasil \\ 2Department of Biochemistry, Centro di Ricerca Interdipartimentale Biotecnologie Innovative, University \\ of Padova, Padova, PD, Italy
}

Correspondence to: F. Langone, Departamento de Fisiologia e Biofísica, Instituto de Biologia, UNICAMP, 13083-970 Campinas, SP, Brasil

Fax: +55-19-3521-6185. E-mail: langone@unicamp.br

\begin{abstract}
Ciliary neurotrophic factor (CNTF) is a cytokine that plays a neuroprotective role in relation to axotomized motoneurons. We determined the effect of daily subcutaneous doses of CNTF $(1.2 \mu \mathrm{g} / \mathrm{g}$ for 5 days; $\mathrm{N}=13)$ or PBS $(\mathrm{N}=13)$ on the levels of mRNA for $\mathrm{Bcl}-2$ and $\mathrm{Bax}$, as well as the expression and inter-association of $\mathrm{Bcl}-2$ and $\mathrm{Bax}$ proteins, and the survival of motoneurons in the spinal cord lumbar enlargement of 2-day-old Wistar rats after sciatic nerve transection. Five days after transection, the effects were evaluated on histological and molecular levels using Nissl staining, immunoprecipitation, Western blot analysis, and reverse transcriptase-polymerase chain reaction. The motoneuron survival ratio, defined as the ratio between the number of motoneurons counted on the lesioned side vs those on the unlesioned side, was calculated. This ratio was $0.77 \pm 0.02$ for CNTF-treated rats vs $0.53 \pm 0.02$ for the PBS-treated controls $(P<0.001)$. Treatment with CNTF modified the level of mRNA, with the expression of Bax RNA decreasing 18\% (with a consequent decrease in the level of Bax protein), while the expression of Bcl2 RNA was increased $87 \%$, although the level of Bcl-2 protein was unchanged. The amount of Bcl-2/Bax heterodimer increased $91 \%$ over that found in the PBS-treated controls. These data show, for the first time, that the neuroprotective effect of CNTF on neonatal rat axotomized motoneurons is associated with a reduction in free Bax, due to the inhibition of Bax expression, as well as increased $\mathrm{Bcl}-2 / \mathrm{Bax}$ heterodimerization. Thus, the neuroprotective action of the CNTF on axotomized motoneurons can be related to the inhibition of this apoptotic pathway.
\end{abstract}

Key words: Ciliary neurotrophic factor; Bax; Neonatal rats; Axotomy; Motoneuron; Immunoprecipitation

*These authors contributed equally to this study.

Research supported by FAPESP (\#05/54613-7) and CNPq (\#473909/2004-9). A.C.S. Rezende, A.S. Vieira and L.F. Rezende are recipients of scholarships from FAPESP (\#04/14210-8, \#05/51335-6, and \#04/09786-8, respectively).

Received May 17, 2007. Accepted October 30, 2008

The sectioning of peripheral nerves in neonatal rodents causes neuronal death by apoptosis and a very massive loss of axotomized motoneurons (1). Such cellular loss has been related to the interruption of trophic support provided by target cells (1). However, the exog- enous administration of neurotrophic molecules can prevent the death of neurons after peripheral nerve sectioning during the neonatal period (2-4). The ciliary neurotrophic factor (CNTF), a cytokine produced by Schwann cells, is capable of protecting the axotomized motoneurons when 
administered systemically in neonatal rodents (2-4). This neuroprotective action of CNTF is considered to be due to the inhibition of apoptosis $(4,5)$, while the effectiveness of systemic administration of CNTF may be due to its ability to cross the blood-brain barrier by a saturable transport system (6).

A key step in the apoptotic signaling cascade is the translocation of the Bax protein from cytoplasm to the mitochondrial membrane (7). This protein plays a critical role in apoptosis, as demonstrated by the absence of axotomy-induced death of motoneurons in Bax-knockout mice (8). Moreover, the apoptotic state can be reversed by heterodimerization of Bax by Bcl-2 (7). Although motoneurons can be protected by CNTF, the mechanisms involved in this protection are not understood. It has been suggested that this effect might be due to changes in the level of $\mathrm{Bax}$ and $\mathrm{Bcl}-2$ expression (7), but it may also be due to the rate of association between these proteins. To our knowledge, the latter hypothesis has not been tested in an experimental model of motoneuron death. Thus, we investigated the effects of exogenous CNTF administration on the expression and interaction of Bax and Bcl-2 in the lumbar spinal cord of neonatal rats after sciatic nerve transection.

Two-day-old Wistar rats received CNTF $(1.2 \mu \mathrm{g} / \mathrm{g}, s c ; \mathrm{N}$ $=13$ ) or PBS ( $N=13), 30$ min before they were anesthetized by hypothermia through immersion in crushed ice for 3-5 min. The left sciatic nerve was then cut at the mid-thigh level. Approximately $3 \mathrm{~mm}$ of the distal stump was removed and the skin was sutured (Silk 8-0, Ethicon, USA). A second dose of CNTF or PBS was then administered, and the rats were allowed to recover from the anesthesia under an incandescent lamp. A third dose was administered $2 \mathrm{~h}$ later, and they were returned to their mothers. Daily subcutaneous injections of CNTF or PBS were then administered during the 4 subsequent days and the rats were sacrificed $24 \mathrm{~h}$ after the last dose, at age P7. All animal procedures were approved by the Animal Care Committee of the Universidade Estadual de Campinas (\#509-1).

Changes in Bax or Bcl-2 gene expression and protein levels were analyzed using semi-quantitative reverse transcriptase-polymerase chain reaction (RT-PCR) and Western blot techniques, respectively. For gene expression analysis, the total RNA was extracted from the frozen lumbar spinal cords of 4 rats from each group using Trizol reagent according to the instructions of the manufacturer (Invitrogen, USA). This mRNA was reverse-transcribed using random primers (Gibco/BRL, USA). The resulting cDNA was amplified using oligonucleotides complementary to the sequences of the Bcl-2 gene (GenBank accession \#L14680; sense GTATGATAACCGGGAGATCG, anti-sense AGCCAGGAG
AAATCAAACAG; $612 \mathrm{bp}$ ) and of the Bax gene (GenBank accession\#U49729; sense AAGAAGCTGAGCGAGTGTCT, anti-sense CAAAGATGGTCACTGTCTGC; 361 bp). As an internal control, RPS29 (ribosomal protein S29; GenBank accession \#NM012876; sense AGGCAAGATGGGT CACCAGC, anti-sense CGACCTGAATGGATGATTCGACT; $201 \mathrm{bp}$ ) was used. The RT-PCR was performed according to standard procedures. The number of cycles was selected from the exponential range for each primer pair. The PCR products were separated on $1.5 \%$ agarose gels and stained with ethidium bromide. All PCR reactions included a negative control. Results are reported as the ratio between the relative optical intensities of the target gene and the internal control product (RPS29). Since the RT-PCR analysis was only semi-quantitative, it was complemented by a Western blot analysis.

For the Western blot analysis, 4 more of the rats from each group were anesthetized by hypothermia on the fifth day post-axotomy, and killed by decapitation. The lumbar spinal cord was rapidly removed, immediately frozen in liquid nitrogen, and stored at $-80^{\circ} \mathrm{C}$. For protein analysis, specimens from each rat were individually homogenized in SDS solubilization buffer (1\% SDS, $50 \mathrm{mM}$ phosphate buffer, pH 7.4, 10 mM EDTA, 5\% $\beta$-mercaptoethanol, and $1 \%$ complete protease inhibitor; Sigma Catalogue \#P8340, USA) at room temperature. Homogenates were incubated at $95^{\circ} \mathrm{C}$ for $20 \mathrm{~min}$, and insoluble material was then removed by centrifugation $(20 \mathrm{~min}$ at $12,000 \mathrm{~g}$ ). The protein concentration in the supernatant was determined by the Bradford dye binding method. SDS polyacrylamide gel electrophoresis and electro-blotting to nitrocellulose were carried out according to standard techniques. The resulting membranes were blocked with nonfat dry milk and then incubated with antibodies for Bax (1:500, sc-493, Santa Cruz, USA). Immunoreactive bands were detected by chemiluminescence according to manufacturer instructions (Super Signal West Pico, Thermo Scientific Pierce Protein Research Products, USA). Membranes were also stripped and re-probed with antibodies for Bcl-2 (1:500, sc-492, Santa Cruz); $\beta$-actin (1:500, Catalogue \#1.378.996, Boehringer, Germany) was used as an internal control, with results reported as the ratio between the relative absorbance of this control and the Bax or Bcl-2 and $\beta$-actin of the two proteins.

To assess the influence of CNTF treatment on the rate of association between $\mathrm{Bcl}-2$ and Bax, immunoprecipitation was carried out using Western blot analysis. The lumbar spinal cords from CNTF- and PBS-treated rats were harvested as described above. Specimens from each rat were homogenized individually in solubilization buffer at $4{ }^{\circ} \mathrm{C}(0.5 \%$ Triton $\mathrm{X}-100,50 \mathrm{mM}$ phosphate buffer, $\mathrm{pH}$ 
7.4, $10 \mathrm{mM}$ EDTA, $142.5 \mathrm{mM} \mathrm{KCl}$ and $1 \%$ complete protease inhibitor) using a Polytron PTA 2100 apparatus (Kinematica AG, Switzerland) for $20 \mathrm{~s}$ at maximum speed. Insoluble material was removed by centrifugation (20 min at $12,000 \mathrm{~g}$ at $4^{\circ} \mathrm{C}$ ). The protein concentration of the supernatant was determined by the Bradford dye binding method. Aliquots of the protein extracts containing $300 \mu \mathrm{g}$ total protein were incubated overnight at $4^{\circ} \mathrm{C}$ with $10 \mu \mathrm{L}$ rabbit polyclonal antibody for Bcl-2 (sc-492, Santa Cruz). Immunoprecipitates were captured with protein Asepharose (Amersham, USA) for $2 \mathrm{~h}$ and washed once in the solubilization buffer, followed by washing in the same buffer without Triton X-100. Immunoprecipitates were then solubilized with $30 \mu \mathrm{L}$ SDS-PAGE loading buffer, heated in a water bath at $95^{\circ} \mathrm{C}$ for $5 \mathrm{~min}$, electrophoresed through $12 \%$ SDS-PAGE gels and transferred to nitrocellulose membranes according to standard techniques. The membranes were blocked with nonfat dry milk and then incubated with antibodies for Bax (1:500, sc-493, Santa Cruz). Immunoreactive bands were detected by chemiluminescence according to the manufacturer instructions of the Super Signal West Pico (Thermo Scientific Pierce Protein Research Products). The membranes were then stripped and re-probed with antibodies for Bcl-2 (1:500, sc-492, Santa Cruz). Results are reported as the ratio between relative absorbance of the Bax and Bcl-2.

The results of the RT-PCR, Western blot and immunoprecipitation analyses were evaluated using the Student $t$ test, and significance was set at a $95 \%$ confidence level.

In order to confirm CNTF-mediated motoneuron protection, the remaining 5 rats of each group were killed by perfusion through the heart with paraformaldehyde on the 5 th day post-axotomy. The lumbar spinal cord was dissected and processed for paraffin embedding. Transverse semi-serial sections $(8 \mu \mathrm{m})$ were made and stained with cresyl violet (Sigma). For each rat, 20 serial sections of the L4 level of the spinal cord were counted (every fourth section). All motoneurons of the ventrolateral group (lamina IX) containing a clearly visible nucleolus in the section plane were counted. The unlesioned side of the spinal cord was used as a control. Motoneuron survival ratio, defined as the ratio between the number of motoneurons counted on the lesioned side vs those on the unlesioned side (9), was evaluated using the Student $t$-test, with significance considered at a $95 \%$ confidence level. The motoneuron survival ratio was higher in CNTF-treated rats than in PBS-treated rats $(0.77 \pm 0.02$ vs $0.53 \pm 0.02$, respectively; $P<0.001)$.

Rats receiving CNTF treatment exhibited modification in the level of mRNA (Figure 1), with that for Bax expression decreasing 18\% (Figure 1A), while that for Bcl-2 expression increased $87 \%$ (Figure 1B). The level of Bax protein accom- panied the decrease in mRNA expression (Figure 2A), although the level of $\mathrm{Bcl}-2$ protein was unaffected by the increase in gene expression (Figure $2 \mathrm{~B}$ ). Despite the reduction in the level of Bax, the rate of association of this protein with $\mathrm{Bcl}-2$ increased (Figure 2C).

In the present paper, we have shown for the first time that CNTF treatment increases the rate of association between Bax and Bcl-2, reducing the amount of free Bax in the cytoplasm. Taken together with the reduction in Bax protein and its mRNA, these data suggest a significant effect of CNTF in reducing the pro-apoptotic action of Bax. Consequently, either Bax protein reduction and/or the increase in $\mathrm{Bcl}-2 / \mathrm{Bax}$ association could be responsible, at least in part, for the CNTF reduction of motoneuron death. These hypotheses are supported by the fact that Bax is essential for axotomy-induced death of these cells in neonatal rodents, as demonstrated in Bax-knockout mice.
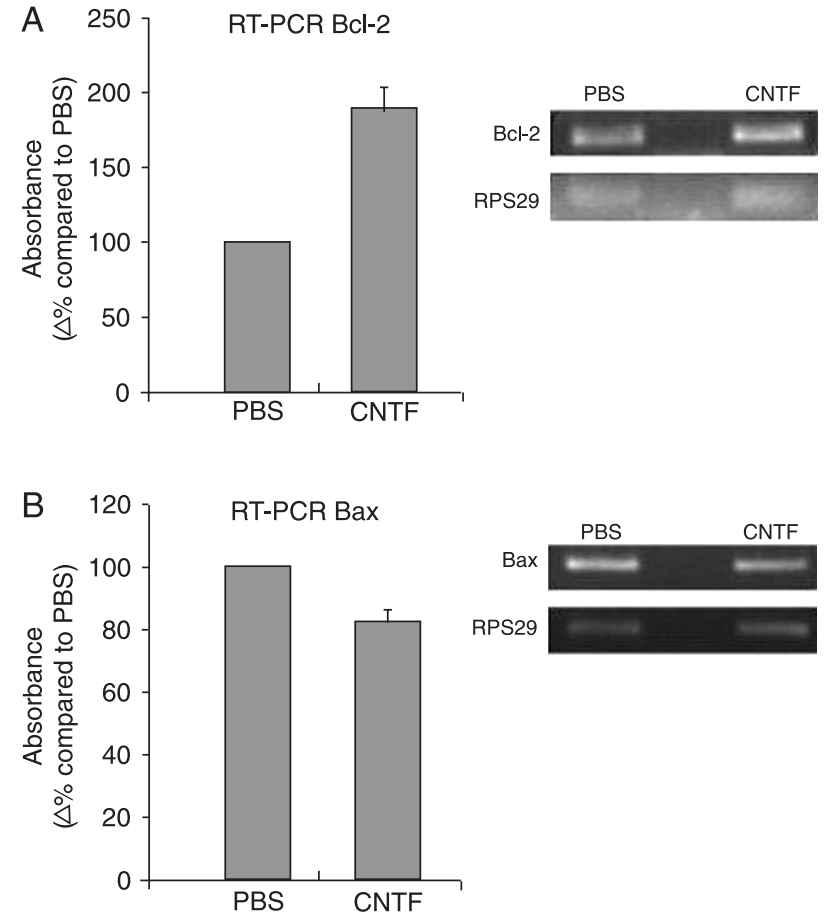

Figure 1. Effects of ciliary neutrophic factor (CNTF) treatment on $\mathrm{Bcl}-2$ (A) and Bax (B) gene expression in lumbar spinal cord of CNTF- and phosphate-buffered saline (PBS)-treated rats. Gels were photographed and the relative absorbance of the target genes (Bax or $\mathrm{Bcl}-2$ ) in contrast with the equivalent and internal control (ribosomal protein S29, RPS29) is reported as mean \pm SEM. The reverse transcriptase-polymerase chain reaction (RTPCR) for Bax showed that treatment with CNTF decreased mRNA expression in relation to that of PBS-treated rats $(\mathrm{P}<$ $0.05)$. The reaction for $\mathrm{Bcl}-2$ showed higher mRNA expression in CNTF-treated rats than in PBS-treated rats $(P<0.05)$. All experiments were done in triplicate and the data were evaluated using the Student $t$-test. 
Indeed, Kinugasa et al. (8) have reported that sciatic nerve section of Bax-deficient neonatal mice (Bax-/-) produced significantly less motoneuron death than did this procedure in wild-type animals (Bax $+/+)$. Moreover, in addition to the reduction in Bax levels, $\mathrm{Bcl}-2$ interacts with $\mathrm{Bax}$ on the mitochondrial membrane, inhibiting pore formation by Bax oligomerization (10).

The importance of $\mathrm{Bcl}-2$ can be seen in the research of
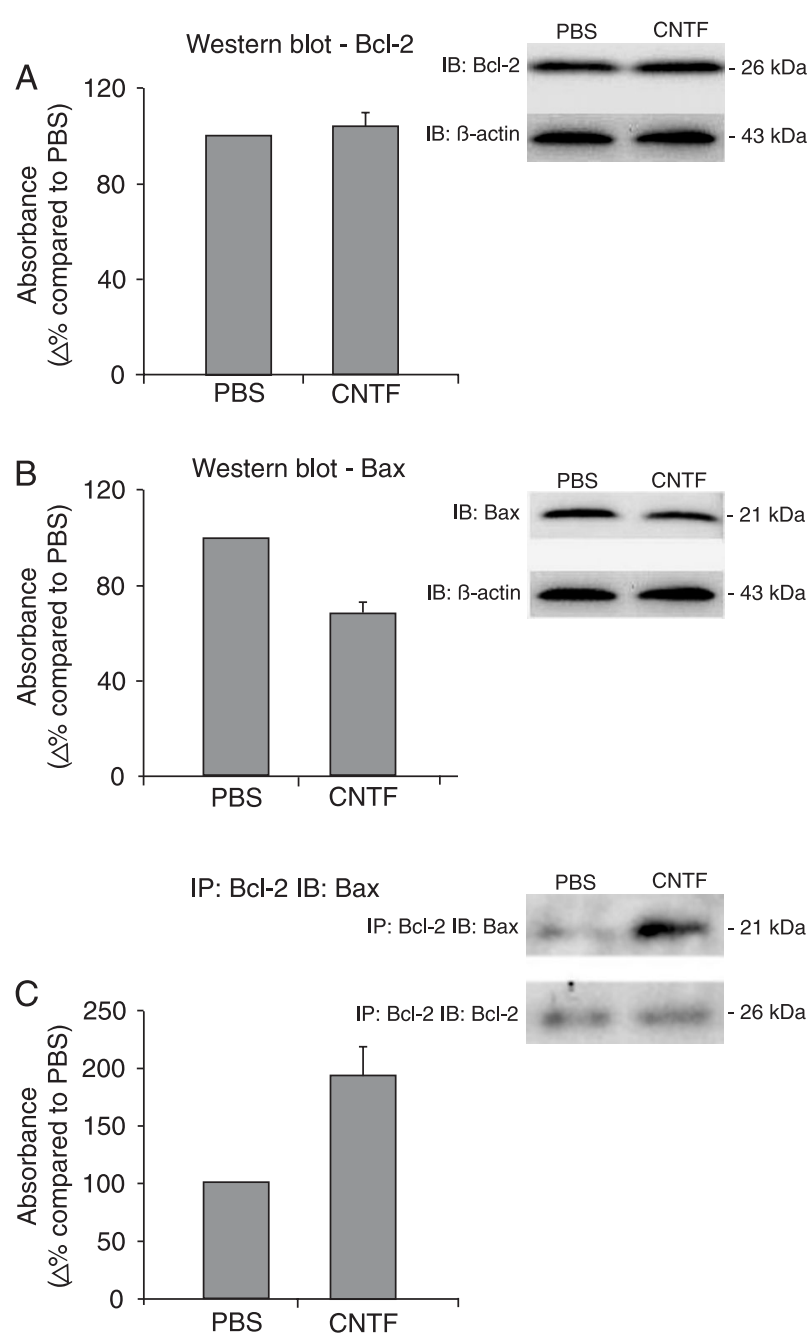

Figure 2. Effects of ciliary neutrophic factor (CNTF) treatment on $\mathrm{Bcl}-2$ (A) and Bax (B) protein expression and ratio of Bcl-2/Bax association (C). The relative absorbance of the 4 samples compared to that of the equivalent $\beta$-actin control is reported as mean \pm SEM for both CNTF- and phosphate-buffered saline (PBS)treated rats. Immunoblot test (IB) for Bax showed a decreased expression of this protein in CNTF-treated rats compared with PBS-treated rats $(P<0.05)$. Bcl-2 immunoprecipitation (IP) followed by IB for Bax showed a larger amount of Bax associated with Bcl-2 in CNTF-treated rats than in those treated with PBS $(\mathrm{P}<$ 0.05). The data were evaluated using the Student $t$-test.
Dubois-Dauphin et al. (11), who observed that motoneurons of the facial nerve of transgenic mice overexpressing human bcl-2 proto-oncogene were resistant to axotomy performed at 2 days of age. These authors showed that the surviving motoneurons exhibited more intense immunostaining for $\mathrm{Bcl}-2$ than did those of the contralateral side 7 days after lesion. Further evidence of the anti-apoptotic role of Bcl-2 was provided by the absence of TUNELlabeled cells from $8 \mathrm{~h}$ to 5 days after facial nerve section (12). However, in the current study, Western blot data did not indicate changes in the level of $\mathrm{Bcl}-2$ protein despite the up-regulation of its mRNA due to CNTF administration. This discrepancy may be explained by the fact that both $\mathrm{Bcl}-2$ level and function can be regulated by proteolytic processing $(7,13)$.

Our observations are consistent with those of Maier et al. (5) who observed that exogenous CNTF protected retinal ganglion cells from death in an acute autoimmune optic neuritis model by increasing $\mathrm{Bcl}-2$ expression and reducing Bax synthesis in these cells. Ours is the first study on the effect of exogenous CNTF on Bcl-2 and Bax expression in spinal motoneurons.

However, it should be taken into consideration that our Western blot, immunoprecipitation and RT-PCR results were obtained from lumbar spinal cord homogenates. Therefore, we cannot exclude the possibility that CNTF may have exerted similar effects on apoptosis-related proteins in other cell types in the lumbar spinal cord, which are or may be indirectly involved in motoneuron survival. The local application of CNTF to the proximal sciatic nerve stump of newborn rats protected the interneurons, which may help preserve the integrity of the motoneurons (4). Moreover, the interaction of exogenous CNTF with the increased CNTF receptor $\alpha$ resulting from astrocyte stimulation by peripheral axotomy may also contribute to motoneuron survival (14). It has also been demonstrated that the administration of CNTF enhances the production of neurotrophic factors by astrocytes, thus promoting the release of fibroblast growth factor-2 (15), which is capable of increasing Bcl-2 while simultaneously decreasing Bax in retinal ganglion cells (16).

Our results show that CNTF exerts its neuroprotective action on immature spinal motoneurons, at least in part, by changing the intracellular balance of $\mathrm{Bax} / \mathrm{Bcl}-2$ in the lumbar spinal cord of neonatal rats after sciatic transection.

\section{Acknowledgments}

The authors would like to thank C.V. Ferreira for the donation of the anti-Bax antibody and Linda Gentry ElDash for the linguistic review of the manuscript. 


\section{References}

1. Lawson SJ, Lowrie MB. The role of apoptosis and excitotoxicity in the death of spinal motoneurons and interneurons after neonatal nerve injury. Neuroscience 1998; 87: 337348.

2. Ikeda K, Iwasaki Y, Shiojima T, Kinoshita M. Neuroprotective effect of various cytokines on developing spinal motoneurons following axotomy. J Neurol Sci 1996; 135: 109113.

3. Sendtner M, Kreutzberg GW, Thoenen H. Ciliary neurotrophic factor prevents the degeneration of motor neurons after axotomy. Nature 1990; 345: 440-441.

4. Oliveira AL, Risling M, Negro A, Langone F, Cullheim S. Apoptosis of spinal interneurons induced by sciatic nerve axotomy in the neonatal rat is counteracted by nerve growth factor and ciliary neurotrophic factor. J Comp Neurol 2002; 447: 381-393.

5. Maier K, Rau CR, Storch MK, Sattler MB, Demmer I, Weissert $R$, et al. Ciliary neurotrophic factor protects retinal ganglion cells from secondary cell death during acute autoimmune optic neuritis in rats. Brain Pathol 2004; 14: 378387.

6. Pan W, Kastin AJ, Maness LM, Brennan JM. Saturable entry of ciliary neurotrophic factor into brain. Neurosci Lett 1999; 263: 69-71.

7. Adams JM, Cory S. The Bcl-2 protein family: arbiters of cell survival. Science 1998; 281: 1322-1326.

8. Kinugasa T, Ozaki S, Hamanaka S, Kudo N. The effects of sciatic nerve axotomy on spinal motoneurons in neonatal Bax-deficient mice. Neurosci Res 2002; 44: 439-446.

9. Rogerio F, de Souza QL, Teixeira SA, Oliveira AL, de Nucci G, Langone F. Neuroprotective action of melatonin on neonatal rat motoneurons after sciatic nerve transection. Brain
Res 2002; 926: 33-41.

10. Dlugosz PJ, Billen LP, Annis MG, Zhu W, Zhang Z, Lin J, et al. Bcl-2 changes conformation to inhibit Bax oligomerization. EMBO J 2006; 25: 2287-2296.

11. Dubois-Dauphin $M$, Frankowski $H$, Tsujimoto $Y$, Huarte J, Martinou JC. Neonatal motoneurons overexpressing the bcl-2 protooncogene in transgenic mice are protected from axotomy-induced cell death. Proc Natl Acad Sci U S A 1994; 91: 3309-3313.

12. de Bilbao F, Dubois-Dauphin M. Time course of axotomyinduced apoptotic cell death in facial motoneurons of neonatal wild type and bcl-2 transgenic mice. Neuroscience 1996; 71: 1111-1119.

13. Ojala PM, Yamamoto K, Castanos-Velez E, Biberfeld $P$, Korsmeyer SJ, Makela TP. The apoptotic v-cyclin-CDK6 complex phosphorylates and inactivates Bcl-2. Nat Cell Biol 2000; 2: 819-825.

14. Kirsch M, Schneider T, Lee MY, Hofmann HD. Lesioninduced changes in the expression of ciliary neurotrophic factor and its receptor in rat optic nerve. Glia 1998; 23: 239248.

15. Albrecht PJ, Dahl JP, Stoltzfus OK, Levenson R, Levison SW. Ciliary neurotrophic factor activates spinal cord astrocytes, stimulating their production and release of fibroblast growth factor-2, to increase motor neuron survival. Exp Neurol 2002; 173: 46-62.

16. Rios-Munoz W, Soto I, Duprey-Diaz MV, Blagburn J, Blanco RE. Fibroblast growth factor 2 applied to the optic nerve after axotomy increases Bcl-2 and decreases Bax in ganglion cells by activating the extracellular signal-regulated kinase signaling pathway. J Neurochem 2005; 93: 14221433. 